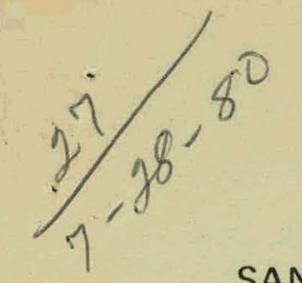

SAND80-0655

Unlimited Release

UC-32

\title{
An Interval Analysis Approach to Rank Determination in Linear Least Squares Problems
}

(Proposed for Submission to SIAM Journal of Scientific and Statistical Computing)

Thomas A. Manteuffel

Prepared by Sandia Laboratories, Albuquerque, New Mexico 87185

and Livermore, California 94550 for the United States Department

of Energy under Contract DE ACO4-76DP00789

Printed June 1980

\section{Sandia National Laboratories}




\section{DISCLAIMER}

This report was prepared as an account of work sponsored by an agency of the United States Government. Neither the United States Government nor any agency Thereof, nor any of their employees, makes any warranty, express or implied, or assumes any legal liability or responsibility for the accuracy, completeness, or usefulness of any information, apparatus, product, or process disclosed, or represents that its use would not infringe privately owned rights. Reference herein to any specific commercial product, process, or service by trade name, trademark, manufacturer, or otherwise does not necessarily constitute or imply its endorsement, recommendation, or favoring by the United States Government or any agency thereof. The views and opinions of authors expressed herein do not necessarily state or reflect those of the United States Government or any agency thereof. 


\section{DISCLAIMER}

Portions of this document may be illegible in electronic image products. Images are produced from the best available original document. 
Issued by Sandia Laboratories, operated for the United States Department of Energy by Sandia Corporation.

\section{NOTICE}

This report was prepared as an account of work sponsored by the United States Government. Neither the United States nor the Department of Energy, nor any of their employees, nor any of their contractors, subcontractors, or their employees, makes any warranty, express or implied, or assumes any legal liability or responsibility for the accuracy, completeness or usefulness of any information, apparatus, product or process disclosed, or represents that its use would not infringe privately owned rights. 


\author{
SAND80-0655 \\ An Interval Analysis Approach to Rank \\ Determination in Linear Least Squares Problems \\ Unlimited Distribution
}

Thomas A. Manteuffel

Numerical Mathematics Div. 5642

Sandia National Laboratories

Albuquerque, NM 87185

The linear least squares problem $A \underline{x} \cong \underline{b}$ has a unique solution only if the matrix $A$ has full column rank. Numerical rank determination is difficult, especially in the presence of uncertainties in the elements of A. This paper proposes an interval analys is approach. We define a set of matrices $A$ that contains all possible perturbations of $A$ due to uncertainties and say that $A^{I}$ is rank deficient if any. member of $\mathrm{A}$ is rank deftcient. A modification to the Q-R decomposition method of solution of the least squares problem allows a determination of the rank of $A^{I}$, and a partial interval analysis of the solution vector $x$. This procedure requires the computation of $R^{-1}$. Another modification is proposed which determines the rank of $\mathrm{A}^{\mathrm{I}}$ without computing $R^{-1}$. The additional computational effort is $O\left(N^{2}\right)$ where $N$ is the column dimension of $A$. 
1. Introduction

The linear least squares problem

$(1.1)$

$A \underline{x} \cong \underline{b}$

has a unique solution only if the matrix $A$ is of full column rank. Numerical determination of rank is not always easy, especially in light of uncertaintles in the elements of $A$. It is of ten the case that $A=\left(a_{1 j}\right)$ is known to be correct only within certain tolerances and the desired but unknown $\hat{A}=\left(\hat{a}_{1 j}\right)$ satisfies

$$
\hat{a}_{1 j}=a_{1 j}+\psi_{1 j},\left|\psi_{1 j}\right|<\max \left(\gamma_{1 j}\left|a_{1 j}\right|, \delta_{1 j}\right)
$$

where $\gamma_{1 f}, \delta_{1 f}$ are relative and absolute error bounds. The uncertainty in $\hat{A}$ may cause rather large uncertainties in the solution, $x$. If any $\hat{A}$ in (1.2) is rank deficlent, then a numerical solution may be completely incorrect.

It is essential that algorithms designed to solve linear least squares problems address the question of rank deficiency. A complete answer would require an extensive interval analysis and the arrays of bounds $\left(\gamma_{1 j}\right)$ and $\left(\delta_{1 j}\right)$ (cf. Hanson and Smith, [7], [8]). For the most part this extra computation is unwarranted. In this paper we address the sltuation where the matrix is assumed to be of full rank. The most efficient algorithm for this case is the $Q R$ decomposition of A based upon Householder reflections (Golub [5]). We will show that a minor modification of this algorithm will yield bounds that help determine the rank of the numerically uncertain matrix A. 
The algorithms proposed here yield as much information about the possible rank deficlency of. A as a singular value decomposition (Golub and Kahan [4]). The computational cost of a singular value decomposition is difficult to predict because of the iterative part of the algorithm (cf. Lawson and Hanson, page 122). As a general rule, the total cost is 2 to 8 times that of the fixed part of the algorithm. The algorithms proposed here are less costly than even the fixed portion of the singular value decomposition.

\subsection{Notation}

We will use the following notation. Capital letters will denote matrices, underlined small letters will denote vectors, and small letters will denote scalers. The $M \times N(M>N)$ real valued matrix

$$
A=\left(a_{i j}\right)=\left(\begin{array}{cccc}
1 & 1 & & 1 \\
a_{1} & \underline{a}_{2} & \cdots & \underline{a}_{N} \\
1 & 1 & & 1
\end{array}\right)
$$

has a singular value decomposition

$$
A=U \Sigma V^{*} ; \Sigma=\operatorname{diag}\left(\sigma_{1}, \ldots, \sigma_{N}\right), 0<\sigma_{N}<\ldots \leqslant \sigma_{1},
$$

where $\mathrm{U}_{\mathrm{MxN}}$ and $\mathrm{V}_{\mathrm{NxN}}$ are unitary matrices. We will use the vector norms

$$
\begin{aligned}
& \|\underline{v}\|_{\infty}=\max _{i}\left|v_{1}\right| \\
& \|\underline{v}\|_{1}=\sum_{i}\left|v_{i}\right| \\
& \left.\|\underline{v}\|_{2}=\underset{i}{\sum}\left|v_{i}\right|^{2}\right)^{1 / 2}
\end{aligned}
$$

and matrix norms 


$$
\begin{aligned}
& \|A\|_{\infty}=\max _{1} \sum\left|a_{i j}\right| \\
& \|A\|_{1}=\max _{j} \sum_{1}\left|a_{1 j}\right|=\max _{j}\left\|\underline{a}_{j}\right\|_{1} \\
& \|A\|_{2}=\max _{\|\underline{x}\|_{2}=1} \quad\|\underline{A x}\|_{2}=\sigma_{1}
\end{aligned}
$$

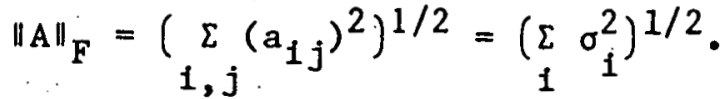

The 2-norm may appear without oubscript. The verinr ef will donotc the $j^{\text {th }}$ basis vector and $\underline{e}=(1,1, \ldots, 1)^{\mathrm{T}}$. We will let

$$
C_{1}(A)=\|A\|_{1}\left\|A^{+}\right\|_{1} \text {, }
$$

where $A^{+}$is the pseudo-inverse of $A$, be the condition of $A$ in the appropriate norm.

\subsection{Problem Definltion}

Consider the set of matrices that includes all perturbations in (1.2): Let

$$
\text { (1.3) } A_{0}^{I}=\left\{A+\Psi:\left|\underline{\mu}_{\perp j}\right| \leqslant \max \left(\gamma_{1 j}\left|a_{i j}\right| ; \delta_{1 j}\right)\right]
$$

We will say that $A_{0}^{I}$ is of full rank if every $\hat{A} \varepsilon A_{0}^{I}$ is of full rank. A subset of columns of $A_{0}^{I}$ will be said to be linearly independent if there exists no $\hat{A} \varepsilon A_{0}^{I}$ whose corresponding columns are linearly dependerit. An independent set of Interval columns will be said. to be maximal if no larger independent set includes it. The Rank ( $A_{0}^{I}$ ) will be the index of the largest maximal independent set of columns of $A_{U^{*}}^{I}$ This yields.

$$
\operatorname{Rank}\left(A_{0}^{I}\right)<\min _{A \varepsilon A_{0}^{I}} \operatorname{Rank}(\hat{A})
$$


Notice that $\left\{\underline{a}_{1}, \underline{a}_{j}\right\}$ and $\left\{\underline{a}_{1}, \underline{a}_{k}^{I}\right\}$ may both be dependent sets, while $\left\{\underline{a}_{j}^{I}, \underline{a}_{k}^{I}\right\}$ may be an Independent set. In fact, one can construct matrices for which the inequality in (1.4) is strict. However, if the right hand side equals $N$, then equality holds.

A desirable result would be to find a largest maximal independent set of columns of $A_{0}^{I}$ and produce an interval solution $\underline{x}^{I}$ that contains all possible solutions based upon this maximal set. Such a result is possible but requires an inordinate amount of computation and storage (cf. Moore [12], Hanson and Smith [7], [8]). The goal of this paper is to obtain as much of this information as possible at a moderate Increase in computation and storage over the Businger-Golub $Q R$ algorithm (Businger and Golub [2]).

Consider a larger interval matrix in which the error bounds are the same throughout each column. Let

$$
\gamma_{j}=\max _{1}\left|\gamma_{i j}\right|, \delta_{j}=\max _{i}\left|\delta_{i j}\right|
$$

(1.5) $\quad A_{1}^{I}=\left\{A+\psi:\left|\psi_{i j}\right|<\max \left(\gamma_{j}\left|a_{i j}\right|, \delta_{j}\right)\right\}$.

One can picture $A_{1}^{I}$ as a matrix each of whose columns is represented. by an M-dimensional box in $R^{M}$ with sides parallel to the axes. The Householder reflections used in the $Q R$ decomposition rotate the vector about the origin. The sides of the box may no longer be paralle1, to the axes. For that reason we enclose the box in a ball. Let

$$
\begin{aligned}
\text { Let } & \varepsilon_{j}=\left(\sum_{1=1}^{M} \max \left(\gamma_{j}\left|a_{1 j}\right|, \delta j\right)^{2}\right)^{1 / 2}, \\
(1.6) \quad A_{2}^{\bar{I}} & =\left\{A+\Psi:\left\|\Psi_{j}\right\|_{2}<\varepsilon_{j}, j=1, \ldots, N\right\} .
\end{aligned}
$$


Finally, consider an interval definition based upon the Frobenius norm: Let

$$
\begin{gathered}
\varepsilon=\left(\sum_{j=1}^{N} \varepsilon_{j}^{2}\right)^{1 / 2}, \\
A_{F}^{I}=\left\{A+\Psi:\|\Psi\|_{F}<\varepsilon\right\} .
\end{gathered}
$$

Th1s is the interval matrix underlying methods based upon singular values. Notice that

$$
A_{0}^{I} \subseteq A_{1}^{I} \subseteq A_{2}^{I} \subseteq A_{F}^{I}
$$

In this paper we will use $A_{2}^{I}$. It has that property that the set of perturbations is invarlant to multiplication by anitary matrix. While rank determination based upon $A_{2}^{I}$ is. not as precise as that based upon $A_{0}^{I}$, it is preferable to rank based upon $A_{F}^{I}$.

Suppose the matrix A has tho QR fnetoriratlul

$$
A=Q R \text {, }
$$

where $R_{M x N}$ is upper trlangular and $Q_{M X M}$ is unitary. If the column order of $A$ is fixed so that the independent columns are ordered first, then the matrix $R$ is unique up to multiplication from the left by a matrix $D=\operatorname{diag}( \pm 1, \ldots, \pm 1)$. Once $R$ is fixed the first $K=\operatorname{Rank}(A)$ columns of $Q$ are unique, while the remaining $M-K$ columns span the null space of . $A^{T}$. Let us define the Interval matrix $R^{I}$ to be the set of upper triangular matrices that result from a $Q R$ decomposition of matrices in $A^{I}$; that is,

$$
R_{2}^{I}=\left\{\hat{R}=R+\Omega: \hat{A}=\hat{Q} \hat{R}, \hat{A} \varepsilon A_{2}^{\prime I}\right\}
$$

Not1ce that

$$
\operatorname{Rank}\left(R_{2}^{I}\right)=\operatorname{Rank}\left(A_{2}^{I}\right)
$$


Statements about the Rank $\left(R_{2}^{I}\right)$ can be thought of as bounds on the size of the diagonal elements of the upper triangular perturbation matrices $\Omega$.

\subsection{Overview}

We will show in Section 2 that while singular value decomposition yields a partial answer to the rank of $A_{2}^{I}$, an equally definitive answer can be garnered from a QR decomposition.and inspection of quantities related to $\left\|R^{-1}\right\|_{1}$. The latter can be established with less computational effort. In Section 3 an algorithm will be proposed that embodies a nearly optimal row and column pivot strategy and determines a maximal set of independent columns of $A_{2}^{I}$. Further, a partial interval analysis of $\underline{x}^{I}$ will be presented. This algorithm requires the construction of $R^{-1}$ as the $Q R$ decomposition proceeds but remalns computationally more efficient than singular value decomposition. In Section 4 it will be shown that a maximal set of columns may be determined without constructing $R^{-1}$. The additional computation over the $Q R$ decomposition is $O\left(N^{2}\right)$. Section 5 will discuss numerical results.

This work is similar in nature to that of Golub, Klema, and Stewart [6]. There the goal is to find an acceptable reduced rank solution based upon $\Lambda_{F}^{I}$. Here we assume full rank and only consider. reduced rank solutions when rank deficlency is detected. No comparison between reduced rank solutions is presented. Their algorithm requires more work and assumes less information about the uncertainties in A.

The bounds established in Section 4 are similar in nature to those of Cline, Moler, Stewart, and Wilkinson [3] and the $O(N)$ bounds of Anderson and Karasalo [1]. These papers deal with lower and upper 
bounds of $C_{1}(R)$ respectively. The bounds of Section 4 also yield upper and lower bounds on $C_{1}(R)$. The lower bound is similar to that of Cline et al. and the upper bound will be better than that of Anderson and Karasalo. Moreover, upper and lower bounds are constructed for each column. These bounds are used to determine the independence of the columns. When the gap between the upper and lower bounds leaves Independence in question, the exact value is computed.

This analysis precludes an accurate rank determination when a weighted least squares solution is sought in which the weights vary greatly in magnitude. Singular value analysis fails here as well. Further study is being conducted in an attempt to incorporate this case (cf. Powell and ReId.[14]).

2. Rank of $A_{2}^{I}$

For convenfence we will first assume that the columns of the matrix A have been scaled so that the uncertainty is the same in each. This does not affect the solution of (1.1). Later we will lift this restriction.

We have

$$
A_{2}^{I}=\left\{A+\Psi:\left\|\Psi_{j}\right\|_{2}<\varepsilon, j=1, \ldots, N\right\},
$$

$$
R_{2}^{I}=\left\{\hat{R}: \hat{A}=\hat{Q} \hat{R}, \hat{A} \in A_{2}^{I}\right\}
$$

Let

$$
\Psi_{2}^{I}=\left\{\Psi:\left\|\Psi_{j}\right\|_{2}<\varepsilon, j=1, \ldots, N\right\}
$$

\subsection{Tests Based On Singular Values}

We have the following well known result: 


\section{Theorem 2.1}

If $\sigma_{k}<\varepsilon$, then $\operatorname{Rank}\left(A_{2}^{I}\right)<k$

If $\sigma_{k}>\sqrt{N}$, then $\operatorname{Rank}\left(A_{2}^{I}\right)>k$

Proof. Notice that if $\psi \in \psi_{2}^{I}$, then $\mid \psi I_{F}<\in \sqrt{N_{0}}$ Further, not1ce that if $\| Y I_{F}<\varepsilon$, then $Y \in \Psi_{2}^{I}$. The theorem follows from well known results on sigular values (cf. Stewart [13], page 320).

Thus, the siggular values will determine the rank deficiency of $A_{2}^{I}$ to within $\sqrt{N}$. In general, the $\sqrt{N}$ cannot be omitted.

\subsection{Tests Based on $\mathrm{R}^{-1}$.}

Rark tests based upon $\left|R^{-1}\right|_{1}$ give similar results. First, let

$$
R^{-1}=\left(\delta_{1 j}\right), p_{j}=\sum_{1=1}^{j}\left|\delta_{1 j}\right| ;
$$

then,

$$
\rho=I R^{-1} I_{1}=\max _{j} \rho_{j}
$$

Theorem 2.2 Let $\left\{\rho_{f_{l}}\right\}_{l=1}^{k}$ be a subset of the $\rho_{j}$ 's.

$$
\operatorname{If}\left(\sum_{\ell=1}^{k} \rho_{J_{\ell}}^{2}\right)^{1 / 2}<\frac{1}{\varepsilon} \text {, then } \operatorname{Rank}\left(A_{2}^{I}\right)>k \text {. }
$$

Proof Let $A=Q R$ be the $Q R$ decomposition of $A$. Assuming $R$ is of full rank, then $\operatorname{Rank}(A+\Psi)=\operatorname{Rank}\left(I+Q * \Psi R^{-1}\right)$. If $k=N$, we have

$$
\begin{aligned}
I \Psi_{R}^{-1} I_{F} & =\left(\sum_{j=1}^{N} \sum_{I=1}^{M} \Psi_{1} \delta_{1 j} I_{2}^{2}\right)^{1 / 2} \\
& <\left(\sum_{j=1}^{N}\left(\sum_{I=1}^{M}\left|\Psi_{1}\right|\left|\delta_{1 j}\right|\right)^{2}\right)^{1 / 2} \\
& <\varepsilon\left(\sum_{j=1}^{N} \rho_{j}^{2}\right)^{1 / 2}<1 .
\end{aligned}
$$

If $k<N$, consider the submatrix of $Y R^{-1}$ consisting of those columns assoclated with the $\rho_{j}$ 's in the subset. Denote this by 
$\left(\Psi R^{-1}\right)_{k} \cdot$ Again we have

$$
\left\|\left(\Psi R^{-1}\right)_{k}\right\|_{F}<1,
$$

which Implies that these $k$ columns are linearly independent for every $\Psi \varepsilon \Psi_{2}^{\mathrm{I}}$. The case in which $\mathrm{R}$ is singular is proved similarly.

The next result ylelds a more convenient form for rank determination.

\section{Theorem 2.3}

If $\rho>\frac{1}{\varepsilon}$, then $A_{2}^{I}$ is rank deficient.

If $\rho<\frac{1}{\varepsilon \sqrt{N}}$, then $A_{2}^{I}$ is of full rank.

Further, if $k=$ index $\left\{\rho_{j}: \rho_{j}<\frac{1}{\varepsilon \sqrt{N}}\right\}$, then Rank $\left(A_{2}^{I}\right)>k$.

Proof If $A=Q R$ is the $Q R$ decomposition of $A$, then the matrix $A=A+\Psi, \Psi \dot{\varepsilon} \Psi_{2}^{I}$, has the same Iinearly independent sets of columns as the matrix $Q^{*}(A+\Psi)=R+\Phi$. Note that $\Phi \varepsilon \Psi I$. Assume $\mu_{k}=\frac{1}{\varepsilon}$ for some $k$. Define $\Psi$ cuch that

$$
\Psi_{1}=-s i g n\left(\delta_{i k}\right) / \rho_{k} Q \underline{e}_{k} \text {. }
$$

We have $\Psi \in \Psi \frac{I}{2}$, and it is easy to show that

$$
(\mathrm{R}+\Phi)\left(\mathrm{R}^{-1} \underline{\underline{e}}_{\mathrm{k}}\right) \equiv \underline{0} \text {. }
$$

Thus, $A_{2}^{I}$ is rank deficient.

The last two results follow from Theorem 2.2.

This result is sharp in that the $\sqrt{\mathrm{N}}$ cannot be omitted. Suppose A is such that $k^{-1}, 0$ and $p_{j}=\frac{1}{\varepsilon \sqrt{N}}, j=1, \ldots, N$. Then let $\Psi$ be such that $\Psi_{1}=\frac{\varepsilon}{\sqrt{N}} Q \underline{e},\left(\underline{e}=\sum_{i=1}^{N} \underline{e}_{1}\right)$. We have $\Psi \varepsilon \Psi^{I}$, and

$$
(R+Q \Psi)\left(R^{-1} \underline{e}\right)=0
$$

Thus, A Is rank deficient. On the other hand, suppose 


$$
\mathbf{R}^{-1}=\rho\left(\begin{array}{ccc}
1 & 1 / 2 & 1 / 3 \\
0 & 1 / 2 & -1 / 3 \\
0 & 0 & 1 / 3
\end{array}\right)
$$

One can show that for $\frac{1}{\varepsilon \sqrt{N}}<\rho<\frac{3}{\varepsilon \sqrt{22}}$ we have Rank $\left(A_{2}^{\dot{I}}\right)=N$.

\subsection{Implicit Scaling}

Consider again the interval matrix (1.5) with perhaps different bounds fọr each column. A singular value analysis without first scaling the columns ylelds no information about $A_{2}^{I}$. This is not a major drawback in that column scaling so that the error is equal in each column is a standard practice. However, this scaling can be handled implicitly when bounds are based upon $\mathrm{R}^{-1}$. As in (2.4), let

$$
\rho_{j}=\sum_{i=1}^{N}\left|\delta_{1 j}\right| \varepsilon_{i}
$$

The fnllowing result is analogous to Theorem 2.3 .

\section{Theorem 2.4}

If $\rho_{j}>1$ for some $j$, then $A_{2}^{I}$ is rank deficient.

If $\rho_{j}<\frac{1}{\sqrt{N}}$ for every $j$, then $A_{2}^{I}$ is of full rank.

Fur Llier, if $k=1$ ndex $\left\{\rho_{j}: \rho_{j}<\frac{1}{\sqrt{N}}\right\}$, then $\operatorname{Rank}\left(A_{2}^{I}\right)>k$.

Proof Suppose $A \varepsilon A_{2}^{I}$. Let $E=\operatorname{diag}\left(\frac{1}{\varepsilon}, \ldots, \frac{1}{\varepsilon_{N}}\right)$; then,

A E $\varepsilon A_{2}^{I}$ with $\varepsilon=\varepsilon_{j}=1, j=1, \ldots, N_{\text {. }}$. We can apply the results of Theorem 2.3 where we now substitute RE for R. Thus; we must find the $\|\cdot\|_{1}$ of the columns of $E^{-1} R^{-1}$. These are the quantities defined in (2.6).

\subsection{Operation Count}

A singular value decomposition involves an iteration and so it Is difficult to accurately predict how much computational effort is 
required. If the Golub algorithm is used (Golub [5]), the fixed computational effort is approximately $2 \mathrm{MN}^{2}$ floating point operations. ${ }^{1}$ If a $Q R$ decomposition is followed by a singular value decomposition, the fixed computation is $\mathrm{MN}^{2}+5 \mathrm{~N}^{3} / 6$ (Lawson and Hanson [10], page 122). The $Q R$ decomposition requires $M N^{2}-N / 3$ operations. Once $R$ is known, the quantities $\rho_{j}$ in (2.4) may be computed by successive back solves requiring $\mathrm{N}^{3} / 6$ operations and $\mathrm{N}$ words of storage. Thus, the $\mathrm{QR}$ algorithm with rank determination based upon $\left\|R^{-1}\right\|_{1}$ is computationally more efficient than singular value decomposition for determining the rank of $\mathrm{A}_{2}^{\mathrm{I}}$ :

There are other advantages to computing $\mathrm{R}^{-1}$. These will be discussed in Section 3. In Section 4 bounds will be developed that yield much of the same information with only $O\left(N^{2}\right)$ extra computation. Section 3

In this section an algorithm will be presented which determines a maximal set of independent columns of $A_{2}^{I}$ (up to a factor of $\sqrt{\mathrm{N}}$ ). Also, bounds on the interval solution $\underline{x}^{I}$ based upon this maximal set are established.

Section 3.1. QR decomposition; row and column pivot strategy.

Consider the QR decomposition of $A$. Using the Golub algorithm (Golub [5]), the upper trlangular matrix $R=A^{(N)}$ is computed in $N$ steps where

$$
A^{(k+1)}=H^{(k)} \underset{r}{P^{(k)}} A^{(k)} P_{C}^{(k)}
$$

$\mathrm{P}_{\mathrm{r}}^{(k)}, \mathrm{P}_{\mathrm{C}}^{(\mathrm{k})}$ are row and column permutation matrices and $\mathrm{H}^{(\mathrm{k})}$ is the Householder reflection matrix that zeros out the subdiagonal elements

1. Here, a floating point operation consists of one addition and one multiplication. 
of the $k+1^{\text {st }}$ column of $\underset{r}{\mathrm{P}^{(k)}} \mathrm{A}^{(k)} \underset{\mathrm{C}}{\mathrm{P}}{ }^{(k)}$. Suppose $\hat{A}=A+\Psi, \Psi \varepsilon \Psi \frac{\mathrm{I}}{2}$ is decomposed using the same row and column pivot order. We have

$$
\hat{R}=R+\Omega
$$

where $\Omega$ is also upper triangular. A consequence of the results in. Manteuffel [11], (Section 4) and Theorem 2.2 yields the bound

$$
\left|\omega_{k k}\right|<\left(\sum_{j=1}^{1} \rho_{j}^{2}\right)^{1 / 2}\left|r_{k k}\right|, k=1, \ldots, N,
$$

where $w_{k k}$ is the $k^{\text {th }}$ diagonal element of $\Omega$ and $\rho_{j}$ is as in (2.6). Also, from the proof of Theorem 2.3 we know that there exists a perturbation such that

$$
\left|\omega_{k k}\right|>\rho_{k}\left|r_{k k}\right|
$$

The vector $\underline{\rho}$ provides bounds on the relative uncertainty of the dlagonal elements of $R$. We would like to choose the column order so that the column with the smallest relative uncertainty comes first (cf. Manteuffel [11], page 31). Notice that if the uncertainty is the same in each column this corresponds to choosing the longest column as In the Businger-Golub algorithm [2]. The choice of the first column affects the size of the uncertainty in the truncated portion of the remalning columns. If one were to rescale at each step so that the truncated portion of the uncertainty were the same for each remaining column, then the algorithms presented here would be similar to that of Businger and Golub. We present an algorithm in which the columns are ordered so that $\rho_{k}$ is as small as possible at step $k$. Also, a row pivot is used so that $H^{(k)}$ reflects onto the nearest basis element. This reduces the growth of the truncated portion of the uncertainty (cf. Manteuffel [11], page 31; Powell and Reid [14]). 


\subsection{Algorithm}

Let us denote

$$
A^{(k)}=\left(\begin{array}{ll}
R_{11}^{(k)} & R_{12}^{(k)} \\
0 & R_{22}^{(k)}
\end{array}\right)
$$

where $R_{11}^{(k)}$ is $k \times k$ and upped triangular. The elements of $R_{11}^{(k)}$ and $\mathrm{R}_{12}^{(\mathrm{k})}$ are fixed (up to column interchanges in $\mathrm{R}_{12}^{(\mathrm{k})}$ ) for step $k+1, \ldots, N$. We can construct the inverse of $R$ as we proced, Cunstier the matrix (3.3)

$$
\Delta^{(k)}=\left(\begin{array}{c}
(k) \\
1 j
\end{array}\right)
$$

where the elements are updated at each step according to the formulas $(3.4)$

$$
\delta_{k k}^{(k)}=\frac{1}{r_{k k}}
$$

$$
\text { for } 1=1, \ldots, k-1
$$

$$
\delta_{i k}^{(k)}=-\delta_{k k}^{(k)} \cdot \delta_{1 k}^{(k-1)}
$$

$$
\begin{aligned}
& \text { for } f=k+1, \ldots, N \\
& \text { for } 1=1, \ldots, k-1
\end{aligned}
$$

$$
\begin{aligned}
& \delta_{1 j}^{(k)}=\delta_{1 j}^{(k-1)}+\delta_{i k}^{(k)} \cdot r_{k j}^{(k)}, \\
& \delta_{k j}^{(k)}=\delta_{k k}^{(k)} r_{k j}^{(k)}
\end{aligned}
$$

We may write

$$
\Delta^{(k)}=\left(\begin{array}{cc}
\Delta(k) & \Delta_{12}^{(k)} \\
11 & \ddots \\
0 & \Delta_{22}^{(k)}
\end{array}\right),
$$

where

$$
\Delta_{11}^{(k)}=\left(R_{11}^{(k)}\right)^{-1}, \Delta_{12}^{(k)}=\left(R_{11}^{(k)}\right)^{-1} R_{12}^{(k)}
$$

and $\Delta_{22}^{(k)}$ is as yet undefined. 
Clearly, after $k$ steps we may compute the quantities $\rho_{j}$, $j m, \ldots, k$. Further, we may examine the columns of $\Delta_{12}^{(k)}$ to deterintne the column permutation that will yield the smallest $\rho_{k+1}$. Let $\underline{s}^{(k)}$ be the vector such that $s_{j}^{(k)}$ contains the \|\|$_{2}$ of the corresponding column of $R_{22}^{(k)}$. For $j=k+1, \ldots, N$ let

$$
\rho_{j}^{(k+1)}=\frac{1}{s_{j}^{(k)}}\left(\varepsilon_{k+1}+\sum_{i=1}^{k}\left|\delta_{i j}^{(k)}\right| \varepsilon_{i}\right) .
$$

If column $j$ were to be chosen to be permuted to the $k+1^{\text {st }}$ position, the resulting $\rho_{k+1}$ would be given by $\rho_{j}^{(k+1)}$. We then choose the column order so that

$$
\rho_{k+1}^{(k+1)}<\rho_{j}^{(k+1)} \quad j=k+1, \ldots, N .
$$

(It should be noted that even though $\rho_{k}$ is chosen to be as small as possible at each step, the inequality $\rho_{k}<\rho_{k+1}$ might not necessarily hold.)

$$
\text { Assume } \rho_{j}<\frac{1}{\sqrt{N}}, j<k \text {, and suppose } \rho_{k+1}=\rho_{k+1}^{(k+1)}<\frac{1}{\sqrt{N}}
$$

By Theorem 2.4 the interval columns $\left\{\underline{a}_{1}^{I}, \ldots, \underline{a}_{k+1}^{I}\right\}$ are 1inearly independent and Rank $\left(A_{2}^{I}\right)>k+1$. On the other hand, suppose that for some $j>k+1$ we have $\rho_{j}^{(k+1)}>1$. By Theorem 2.4 we know that the interval columns $\left\{\underline{a}_{1}^{I}, \ldots . \underline{a}_{k}^{I}, \underline{a}_{j}^{I}\right\}$ are dependent. Therefore, column $j$ may not be used in computing a solution. This column should be ordered last and only the remaining columns need be considered.

Once the $k+1^{s t}$ column has been chosen the rows are permuted so that the element with the largest absolute value in the first column of $R_{22}^{(k)}$ appears at the top of the column. The first column of $R_{22}^{(k)}$ will then be reflected onto the nearest basis element.

In this way a maximal set of independent columns will be constructed. The factor $\sqrt{\mathrm{N}}$ that separates the tests for dependence, and independence 
may have to be dealt with. If $\mathrm{N}$ is not too large, then this factor may be insignificant. Further, since $A_{0}^{I} \subseteq A_{2}^{I}$ by proper inclusion we may feel safe in ignoring the $\sqrt{\mathrm{N}}$. Suppose we construct a set of $\dot{K}_{2}$ columns in this manner. We may then appeal to Theorem 2.2 and determine the largest set of columns, say $K_{1}$ columns, such that $\sum \rho_{j}^{2}<1$. We will then have

$$
K_{1}<\operatorname{rank}\left(A_{2}^{I}\right)<K_{2}
$$

The gap between $k_{1}$ and $k_{2}$ will indicate how precise the rank determination 18. Numerical results show that with few exceptions $k_{1}=k_{2}$ (see Section 5).

The overall cost of this algorithm is $\mathrm{MN}^{2}$ arithmetc operations. The $Q R$ decomposition requires $\mathrm{MN}^{2}-\mathrm{N}^{3} / 3$ operations, construction of $\Delta$ requires $N^{3} / 6$ operations, and the selection of $\rho$ requires $N^{3} / 6$ operations. This is still less than the fixed number of operations required for a singular value decompoeition, which is the swaller of $M N^{2}+5 N^{3} / 3$ or $2 M N^{2}$. Note that $\Delta$ may be constructed over $R$ so that the only extra storage needed is for the vector $\rho$. The extra com putation required to select the column pivot may be unwarranted. In Section 4 upper and lower bounds on $\rho_{k}$ are constructed at a cost of $O\left(N^{2}\right)$ operations and the pivot strategy may be based upon these. In fact, these bounds do not require the construction of $\Delta$. However, $\Delta$ may provide information about $\underline{x}^{I}$, the interval solution.

\section{3 Bounds on Uncertainty}

Once $\Delta=R^{-1}$ has been constructed both $\left\|R^{-1}\right\|_{1}$ and $\left\|R^{-1}\right\|_{\infty}$ may also be computed. The norms $\|R\|_{1}$ and $\|R\|_{\infty}$ can be computed. during the decomposition. With these and the bound

$$
C_{2}(A)<\sqrt{C_{1}(A) C_{\infty}(A)}
$$

One can analyze the relative uncertainty in the various norms; that 
is, the quantity

$$
\frac{\|\underline{x}-\hat{x}\|}{\|\underline{x}\|}
$$

where $\hat{x}$ is the solution of a perturbed system $\hat{A} \underline{\hat{x}}=\underline{\hat{b}}$, can be bounded (cf. Stewart [13], page 223).

We can also look at the term by term uncertainty to bound $\underline{x}^{I}$.

Let $\hat{A}=A+\Psi, \Psi \varepsilon \Psi_{2}^{I}, \underline{\hat{b}}=\underline{b}+\Psi_{N+1},\left\|\Psi_{N+1}\right\|_{2}<\varepsilon_{N+1}$.

If we multiply both equations by $Q$ we have

$$
\begin{aligned}
\underline{R} \underline{x} & =\underline{g} \\
(R+\Phi) \underline{\hat{x}} & =\underline{g}+\underline{f}
\end{aligned}
$$

where $\Phi \varepsilon \Psi_{2}^{I}, \| \underline{f}_{2} \leqslant \varepsilon_{N+1}$. We can write

$$
(\underline{x}-\underline{\hat{x}})=R^{-1}(\underline{f}-\underline{\underline{x}})
$$

and

$$
\begin{aligned}
& \left.\left|x_{1}-\hat{x}_{1}\right|=\underset{k}{\mid \sum} \delta_{i k} f_{k}-\underset{j}{\sum} \underset{k}{\left(\sum \delta_{i k}\right.} \phi_{k j}\right) \hat{x}_{j} \mid \\
& \left.<\sum_{k}\left|\delta_{i k}\right|\left|f_{k}\right|+\sum_{j} \underset{k}{\left(\sum\right.}\left|\delta_{i k}\right|\left|\phi_{k j}\right|\right)\left|\hat{x}_{j}\right| \\
& <\left\|\underline{\delta}_{i}^{T}\right\|_{2}\left(\varepsilon_{N+1}+\sum \varepsilon_{j}\left|\hat{x}_{j}\right|\right)
\end{aligned}
$$

where $\delta_{i}^{T}$ represents the $i^{\text {th }}$ row of $\Delta=R^{-1}$. For small perturbations we may replace $\left|\hat{x}_{j}\right|$ by $\left|x_{j}\right|$ on the right hand side. This interval solution is fairly sharp in that we can always construct a perturbation such that

$$
\left|x_{i}-\hat{x}_{i}\right|>\left\|\delta_{-1}^{T}\right\|_{2}\left(\varepsilon_{N+1}+\left|\sum \varepsilon_{j} \hat{x}_{j}\right|\right) .
$$

of course (3.13) cannot be made to hold for all i simultaneously.

\section{4 Reduced Rank Solutions}

Suppose that $A_{2}^{I}$ is found to be rank deficient and that a maximal set of $k<N$ independent columns has been established. Ignoring row and column pivoting, $k$ steps of the standard $Q R$ algorithm would yield a system of the form 
$(3.14) \quad \cdots\left(\begin{array}{ll}\mathrm{R}_{11} & \mathrm{R}_{12} \\ 0 & \mathrm{R}_{22}\end{array}\right)\left(\begin{array}{l}\underline{x}_{1} \\ \underline{x}_{2}\end{array}\right) \cong:\left(\begin{array}{l}\underline{g}_{1} \\ g_{2}\end{array}\right)$

where $R_{11}$ is $k x k$ and upper triangular and $g=Q^{*} \underline{b}$. The truncated least squares solution could be found by setting

$$
\begin{aligned}
& \underline{x}_{1}=R_{11}^{-1} g_{1} \\
& \underline{x}_{2}=\underline{0} .
\end{aligned}
$$

The minimal length least squares solution could be found by finding the minimal length solution to the undetermined system

$$
\left(\begin{array}{ll}
R_{11} & R_{12}
\end{array}\right) \cdot\left(\begin{array}{l}
\underline{x}_{1} \\
\underline{x}_{2}
\end{array}\right)=\underline{g}_{1} .
$$

The algorithm of Section 3.2 produces the matrix

$$
\Delta=\left(\begin{array}{lll}
\Delta_{11} & \Delta_{12} \\
0 & : & R_{22}
\end{array}\right)
$$

where $\Delta_{11}=\mathrm{R}_{11}^{-1}, \Delta_{12}=\mathrm{R}_{11}^{-1} \mathrm{R}_{12}$. The truncated solution can be found using $\Delta$ by setting

$$
\begin{aligned}
& \underline{x}_{1}=\Delta_{11} \underline{g}_{1} \\
& \underline{x}_{2}=\underline{0} .
\end{aligned}
$$

The minfmal length least squares solution is more complicated. Consider multiplying $(3.15)$ by $\Delta_{11}$. We have

$$
\left(\begin{array}{ll}
I & \Delta_{12}
\end{array}\right)\left(\begin{array}{l}
x_{1} \\
x_{2}
\end{array}\right) . \quad=\Delta_{11} g
$$

The minimal length solution to (3.16) can be found by minimizing

$$
\left\|\underline{x}_{1}\right\|_{2}^{2}+\left\|\underline{x}_{2}\right\|_{2}^{2}=\left\|\Delta_{11} \underline{g}_{1}-\Delta_{12} \underline{x}_{2}\right\|_{2}^{2}+\left\|\underline{x}_{2}\right\|_{2}^{2}
$$

This is equivalent to flnding the solution to the least squares problem

$$
\left(\begin{array}{c}
\Delta_{12} \\
-I
\end{array}\right) \underline{x}_{2} \cong\left(\begin{array}{l}
\underline{f} \\
\underline{0}
\end{array}\right)
$$


where $\underline{f}=\Delta_{11} g_{1}$. The residual to (3.17) yields

$$
\left(\begin{array}{l}
\underline{x}_{1} \\
\underline{x}_{2}
\end{array}\right)=\cdots\left(\begin{array}{c}
\frac{f}{\cdots} \\
\underline{0}
\end{array}\right)-\left(\begin{array}{c}
\Delta_{12} \\
-I
\end{array}\right): \underline{x}_{2}
$$

Suppose we construct an orthogonal matrix $\mathrm{H}$ as a product of Householder reflèctions" such that

$$
H\left(\begin{array}{c}
\Delta_{12} \\
-I
\end{array}\right) \quad \therefore\left(\begin{array}{l}
0 \\
U
\end{array}\right)
$$

where $U$ is upper triangular. The residual of $(3.17)$ is that portion of $\left(\begin{array}{l}\underline{f} \\ \underline{0}\end{array}\right)$ that is orthogonal to $\left(\begin{array}{c}\Delta_{12} \\ -1\end{array}\right)^{T}$ or $H *$ times thät portion of $\mathrm{H}\left(\begin{array}{l}\frac{f}{4} \\ 0\end{array}\right)$ that is orthogonal to $\left(\begin{array}{l}0 \\ U\end{array}\right)^{\mathrm{T}}$. We may write

$$
\left(\begin{array}{l}
\underline{x}_{1} \\
\underline{x}_{2}
\end{array}\right)=H *\left(\begin{array}{ll}
I & 0 \\
0 & 0
\end{array}\right) H\left(\begin{array}{l}
\underline{f} \\
\underline{0}
\end{array}\right)
$$

The transformation $H$ can be constructed and stored over. $\Delta_{12}$ and $R_{22}$ Notice that $\Delta_{11}$ remains intact so that both truncated solutions and minimal length solutions can be constructed for future right hand sides.

Section $4 \circ\left(N^{2}\right)$ Bounds

In this section we develop an algorithm that determines a maximal linearly independent set of columns of $A_{2}^{I}$ at an additional cost of $0\left(\mathrm{~N}^{2}\right)$ operations over the $\mathrm{QR}$ decomposition. 4.1 Upper and Lower Bounds a

Let us again assume that the columns of A have been scaled so that $\varepsilon=\varepsilon_{j} ; j=1, \ldots, N$. We will lift this restriction later. Consider $R$ to be an $H$-matrix ${ }^{3}$; then, the corresponding $M$-matrix ${ }^{2}$ is

$$
\bar{R}=\left(\bar{r}_{i j}\right) ; \bar{r}_{i i}=\left|r_{1 i}\right| ; \bar{r}_{i j}=-\left|r_{i j}\right|, i \neq j \cdot
$$


Let us write

$$
\begin{aligned}
& R=D(I-T), D=\operatorname{diag}\left(\bar{r}_{11}, \ldots, r_{N N}\right) \\
& \bar{R}=\bar{D}(I-\bar{T}), \bar{D}=\operatorname{diag}\left(\bar{r}_{11}, \ldots, \bar{r}_{N N}\right)
\end{aligned}
$$

Then,

$$
\begin{aligned}
& \mathrm{R}^{-1}=\left(I+T+\ldots+\mathrm{T}^{\mathrm{N}-1}\right) \mathrm{D}^{-1} \\
& \overline{\mathrm{R}}^{-1}=\left(\mathrm{I}+\overline{\mathrm{T}}+\ldots+\overline{\mathrm{T}}{ }^{N-1}\right) \bar{D}^{-1}
\end{aligned}
$$

from which we see that

$$
\bar{R}-1>\left|R^{-1}\right|>0
$$

If we denote $\pi^{-1}=\left(\delta_{i j}\right)$, then.

$$
\bar{\rho}_{j}=\sum_{i=1}^{j} \bar{\delta}_{1 j}>\sum_{i=1}^{j}\left|\delta_{1 j}\right|=\rho_{j}
$$

Since $R^{-1}>0$, the $\rho_{j}$ 's can be conputed with a single back solve. Let $\bar{p}=\left(\rho_{1}, \ldots, \bar{\rho}_{N}\right)^{\mathrm{T}}$; then, if $\underline{e}=\sum_{i=1}^{N} \underline{e}_{i}$, we have

$$
\overline{\mathrm{R}}^{-\mathrm{T}} \underline{\mathrm{e}}=\overline{\bar{\rho}}
$$

and

$$
\bar{\rho}_{j}=\frac{1}{\left|r_{j j}\right|}\left(1+\sum_{i=1}^{j-1}\left|r_{i j}\right| \bar{\rho}_{i}\right) .
$$

The computation of $\bar{\rho}$ can be carried out during the $Q R$ decomposition and, in fact, $\bar{\rho}_{k}$ will be known after the $k^{\text {th }}$ step of the $Q R$ algortthm.

Consider $(4.6)$ with $R^{-T}$ in place of $\bar{R}^{-T}$. If we let

$$
R^{-T}\left(\begin{array}{c} 
\pm 1 \\
\vdots \\
\ddots \\
\pm 1
\end{array}\right)=\left(\begin{array}{c}
\tilde{\rho}_{1} \\
\vdots \\
\dot{\tilde{\rho}_{N}} \\
\tilde{\rho}_{N}
\end{array}\right)
$$

2. A matrix $A=\left(a_{1 j}\right)$ is an M-matrix, if $a_{i j} \leqslant 0$ for $i \neq j$, $A$ is nonsingular and $A^{-1} \geqslant 0$.

3. A matrix $A=\left(a_{i j}\right)$ is an H-matrix if the matrix $\bar{A}=\left(\bar{a}_{i j}\right)$ with $\bar{a}_{11}=\left|a_{11}\right|, \bar{a}_{i j}=-\left|a_{1 j}\right| i \neq j$ is an M-matr $i x$. 
where the signs are to be chosen later, then we have

$$
\left|\tilde{\rho}_{j}\right|=\left|\sum_{1=1}^{j} \pm \delta_{1 j}\right|<\sum_{i=1}^{j}\left|\delta_{1 j}\right|=\rho_{j}
$$

Since $R^{T}$ is lower triangular, we may write

$$
\mathrm{R}^{\mathrm{T}} \underset{\tilde{\rho}}{\tilde{a}}=\left(\begin{array}{c} 
\pm 1 \\
\cdot \\
\cdot \\
\dot{1}
\end{array}\right)
$$

and choose the sign of the $j^{\text {th }}$ element of the right hand side after $\tilde{\rho}_{i}, i<j$ have been computed. We may write

$$
\tilde{\rho}_{j}=\frac{1}{r_{j j}}\left( \pm 1-\sum_{i=1}^{j-1} r_{i j} \tilde{\rho}_{i}\right) \text {, }
$$

and choose the sign so that $\left|\tilde{\rho}_{j}\right|$ is as large as possible. Again, $\tilde{\rho}_{k}$ will be known after the $k^{\text {th }}$ step of the $Q R$ decomposition. We have then

$$
\left|\tilde{\rho}_{j}\right|<\rho_{j}<\bar{\rho}_{j}, j=1, \therefore, N \text {. }
$$

This yields the followlng corullary. to Theorem 2.3.

\section{Corollary 4.1}

If $\left|\tilde{\rho}_{j}\right|>\frac{1}{\varepsilon}$ for some $j$, then $A_{2}^{I}$ is rank deficient.

If $\overline{\rho_{j}}<\frac{1}{\varepsilon \sqrt{N}}$ for every $j$, then $A_{2}^{I}$ is of full rank.

Further; if $k=$ index $\left\{\bar{\rho}_{j}: \bar{\rho}_{j}<\frac{1}{\varepsilon \sqrt{N}}\right\}$, then Rank $\left(A^{I}\right) \geqslant k$.

The lower bounds $\underline{\tilde{\rho}}$ are computed in much the same manner as certain quantities in Cline, Moler, Stewart and Wilkinson ([3]; page 371). There they are concerned with what corresponds to $\underline{\rho}_{2}$ while here we are concerned with each individual $\left|\tilde{\rho}_{j}\right|$.

Following their development, we can enhance the lower bounds by looking ahead before choosing the sign. Suppose $\tilde{\rho}_{j}, j<k$ have 
been chosen. Each $\tilde{\rho}_{j}, j>k$ is determined by

$$
\begin{aligned}
& r_{j j} \tilde{\rho}_{j}=\left(-r_{1 j} \tilde{\rho}_{1}-\ldots-r_{k-1 j} \tilde{\rho}_{k-1}\right)+ \\
& \therefore \quad\left(-r_{k j} \tilde{\rho}_{k}-\ldots-r_{j-1 j} \tilde{\rho}_{j-1} \pm 1\right)
\end{aligned}
$$

where the right hand side has been split into two parts. The first part is determined once $\tilde{\rho}_{j}, j<k$ have been chosen. Denote this part by $\tilde{\rho}_{j}^{(k-1)}$. The two values of $\rho_{k}$ are

$$
\tilde{\rho}_{k}=\frac{1}{r_{k k}}\left(\tilde{p}_{k}(k-1) \pm 1\right)
$$

We denote these by $\tilde{\rho}_{k}^{+}$and ${\tilde{p_{k}}}_{-}^{-}$We know that both values satisfy.

$$
\begin{gathered}
\left|\tilde{\rho}_{k}^{+}\right|<\rho_{k}, \\
\therefore: \\
\left|\tilde{\rho}_{k}\right|<\rho_{k},
\end{gathered}
$$

and so may choose the larger in absolute value for our lower bound. However, we are still free to choose either $\tilde{\rho}_{k}^{+}$or $\tilde{\rho}_{k}$ for computation of $\rho_{j}^{(k)}, j>k$ as long as we are consistent throughout. Let

$$
\underset{j}{\tilde{\rho}(k)+}=\underset{\rho}{\tilde{\rho}} \underset{j}{(k-1)}-r_{k j} \tilde{\rho}_{k}^{+} .
$$

$$
\tilde{\rho}_{j}^{(k)}=\tilde{\rho}_{j}^{(k+1)}-\dot{r}_{k j} \tilde{\rho}_{k}^{+}
$$

and choose the sign to maximize

$$
\text { (4.16) } \sum_{j>k}|\tilde{\rho}(k)|
$$

There is about twice as much work in this algorithm, but the overall work to compute $\tilde{\rho}$ remains $O\left(N^{2}\right)$. Notice that the 
vector of bounds $\underline{\rho}$ does not necessarily satisfy (4.9) but will satisfy $(4.11)$

\subsection{Implicit Scaling}

As in Section (2.3) these bounds can incorporate implicit scaling. If we again consider $A^{I}$ as in (1.5), then the formulas. corresponding to (4.7); and (4.9) become

$(4: 15)^{\prime}: \bar{\rho}_{j}=\frac{1}{\left|r_{j j}\right|}\left(\varepsilon_{j}+\sum_{i=1}^{j-1}\left|r_{i j}\right| \bar{\rho}_{i}\right)$

$$
\tilde{\rho}_{j}=\frac{1}{r_{j j}}\left( \pm \varepsilon_{j}-\sum_{1=1}^{j-1} r_{i j} \tilde{\rho}_{1}\right)
$$

This ylelds the following corollary to Theorem 2.4:

\section{Corollary 4.2}

If $\left|\tilde{\rho}_{j}\right|>1$ for some $j$, then $A_{2}^{I}$ is rank deficient.

If $\bar{\rho}_{j}<\frac{1}{\sqrt{N}}$, for every $j$, then $A_{2}^{I}$ is of full rank.

Further, if $k=$ index $\left\{\bar{\rho}_{j}: \bar{\rho}_{j}<\frac{1}{\sqrt{N}}\right\}$, then Rank $\left(A_{2}^{I}\right) \geqslant k$.

\subsection{Construction of a Maximal Set}

These bounds may be used in the pivot strategy and to construct a maximal set. Since $\rho_{j}<\bar{\rho}_{j}$ we have, as in (3.1), the bound

$$
\left|\omega_{k k}\right|<\left(\sum_{j=1}^{k} \bar{\rho}_{j}^{2}\right)^{1 / 2}\left|r_{k k}\right|, \quad k=1, \ldots ; N \text {. }
$$

Thuo, we may choose the culumn order to make $\vec{\rho}_{k}$ as small as possible at each step. Suppose we compute $\underline{-}^{(k)}$ as a vector of partial sums as we did for $\tilde{p}^{(k)}$. Let

$$
\rho_{j}^{(0)}=\varepsilon_{j}, \quad j=1, \ldots, N
$$

and at step $k$ update $\underline{\underline{\rho}}^{(k)}$ by the formulas 


$$
\bar{\rho}_{k}^{(k)}=\frac{1}{r_{k k}} \bar{\rho}_{k}^{(k-1)}
$$

for $j=k+1, \ldots, N$

$(4.20)$

$$
\bar{\rho}_{j}^{(k)}=\rho_{j}^{-(k-1)}+\left|r_{k j}\right| \rho_{k}^{-(k)} .
$$

We have then $\bar{\rho}_{k}=\bar{\rho}_{k}^{(k)}$.

Again let $\underline{s}^{(k)}$ be the vector such that $s_{j}^{(k)}$ contains the $\|\cdot\|_{2}$ of the corresponding column of $R_{22}^{(k)}$. If column $f$ were to be shifted to position $k+1$, then $\bar{\rho}_{k+1}$ would have the value (k)

$\frac{\rho_{j}}{(k)}$. The pivot strategy is then to choose column $k+1$ so that s.

$$
\bar{\rho}_{k+1}=\frac{\bar{p}_{k+1}^{(k)}}{s_{k+1}^{(k)}}<\frac{\bar{p}_{j}^{(k)}}{s_{j}^{(k)}}, j=k+1, \ldots, N
$$

Assume $\bar{\rho}_{j}<\frac{1}{\sqrt{N}}, j<k$, and suppose that $\bar{\rho}_{k+1}<\frac{1}{\sqrt{N}}$.

By Corollary 4.2 the interval columns $\left\{a_{1}^{I}, \ldots, a_{k+1}^{I}\right\}$ are linearly independent and Rank $\left(\mathrm{A}^{\mathrm{I}}\right)>\mathrm{k}+1$. On the other hand, suppose for some $j>k+1$ we have $\left|\tilde{\rho}_{j}\right|>1$. By Corollary 4.2 we know that the Interval columns $\left\{a_{1}^{I}, \ldots, a_{k}^{I}, a_{j}^{I}\right\}$ are linearly dependent and column $j$ may not be used in computing a solution. Suppose we have

$$
\bar{\rho}_{k+1} \geq \frac{1}{\sqrt{N}}, \tilde{\rho}_{k+1}<1
$$

Corollary 4.2 yields no information. We must then compute the exact value $\rho_{k+1}$. This can be accomplished by a single back solve. Once $\rho_{k+1}$ has been computed we may appeal to our previous strategy for determining linear dependence (see section 
3.2): Since $\rho_{k+1}$ is a better bound than $\bar{\rho}_{k+1}$ we may set $\rho_{k+1}^{-(k+1)}=\rho_{k+1}$. for computation of $(4.20)$

If $\mathrm{A}_{2}^{\mathrm{I}}$ is assumed to be of full rank; that is, it is not clearly rank deficient from the outset; then the bounds $\bar{\rho}_{k}$ w111 most 11 kely suffice for all but at most a few columns. In some of these columns $\tilde{\rho}_{k}$ may provide enough information. The exact value $\rho_{k}$ will be computed $O(1)$ times for all but the most pathological problems. This yields an $0\left(\mathrm{~N}^{2}\right)$ algorithm that produces a maximal set of independent columns of $A_{2}^{I}$ (up to the factor of $\sqrt{\mathrm{N}}$ ).

5. Numerical Results

In this section we will compare the performance of the three algortthms described above: The algorithm of section 3 that computes $\mathrm{R}^{-1}(\mathrm{Ml})$, the algorithm of Section 4 that creates upper and lower bounds (M2), and the algorithm of Section 4 that chooses the lower bound by looking ahead at partial sums (M3). First, consider the following example that demonstrates the Iimitations of the Businger-Golub algorithm.

Let $s=\sin (\theta), c=\cos (\theta)$,

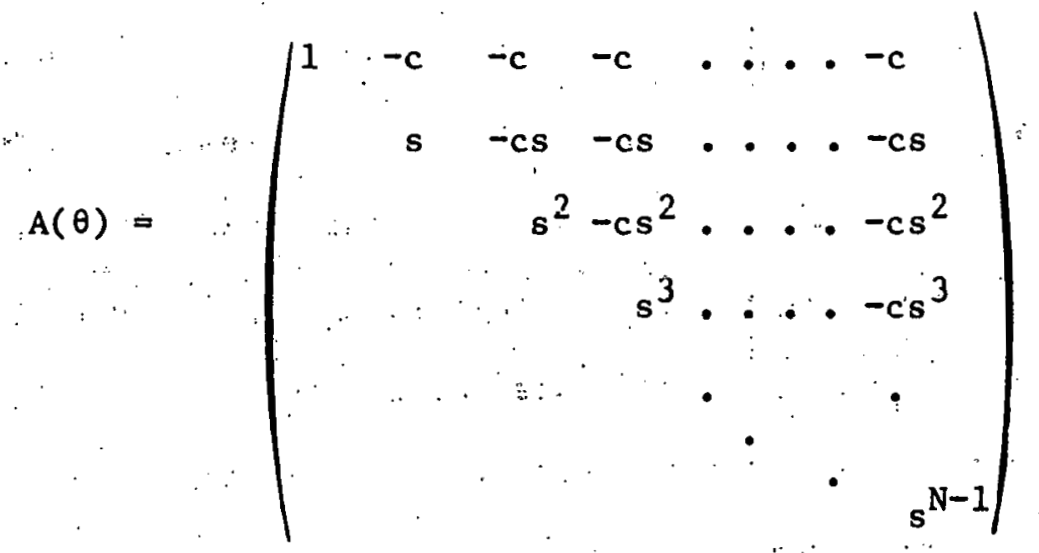

For $0<\theta<\frac{\pi}{2}$ the of diagonal terms remain negative. If we assume that the uncertainty is the same in each column; that is, 


$$
A_{2}^{I}=\left\{A+\Psi:\left\|\Psi_{j}\right\|_{2}<\varepsilon\right\} ;
$$

then $A_{2}^{I}$ is rank deficient for $\varepsilon=\left(\frac{s}{1+c}\right)^{N-1}$. To see this, let $\Psi=-\varepsilon \underline{e}_{N} e^{T}$, and let

Then, we have

$$
\underline{v}=\left(\begin{array}{c}
c(1+c)^{N-2} \\
\vdots \\
\vdots \\
c(1+c) \\
c \\
1
\end{array}\right) ;
$$

$$
(A+\Psi) \underline{v} \equiv \underline{0}
$$

Both the Businger-Golub algorithm and the methods developed above would leave A unchanged. The Businger-Golub algorithm depends upon the size of the diagonal elements for rank determination. With the proper choice of $\theta, \varepsilon$ can be made much smaller than $s^{N-1}$. For example, suppose we let $\varepsilon=10^{-13}, N=53$, and $\theta=58.70^{\circ}$. Then $s^{N-1}=2.807 \times 10^{-4}$, but $A_{2}^{I}$ is rank deficient. To complicate matters $A(\pi-\theta), 0<\theta<\frac{\pi}{2}$, has positive off diagonal elements and is quite well conditioned. The BusingerGolub algorlthm is unable to differentiate between $A(\theta)$ and $A(\pi-\dot{\theta})$.

Ali three of the algorithms presented here properly detect the ranks of $A(\theta)$ and $A(\pi-\theta)$. The method $M 1$ computes $R^{-1}$ and so would be expected to correctly determine rank. For $A(\theta)$ both $M 2$ and $M 3$ compute exact bounds; that is, $\underline{\rho}=\underline{\rho}=\underline{\bar{\rho}}$. For $A(\pi-\theta)$; the lower and upper bounds are quite disparate: $\underline{\underline{\rho}}=\underline{\rho} \ll \underline{\underline{\rho}} . \quad$ In the example cited above, however, only $\rho_{N}$ needed to be computed exactly to determine the rank of $A_{2}^{I}$. 
The ability of the three algorithms to determine numerical rank has been spelled out In the discussion above: However, several questions about their comparative performance must be answered by numerlcal tests.

Methods M2 and M3 will yield essentially the same p1vot order, based upon the upper bounds $\vec{p}$. The difference between $M 2$ and $M 3$ lies in the computation of the lower bounds $\tilde{p}$. While M3 generaliy produces better bounds, the cost of computing those bounds is higher. The advantage to having better bounds 11es in less frequent

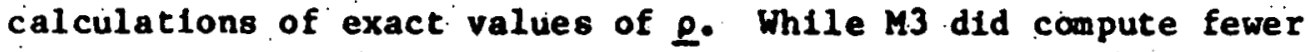
exact values, the difference was not suffictent to warrant the extra arithmetic as w11 be shown below.

In M1 the pivot order is determined by $\underline{\rho}$, and so it may differ from the pivot order produced by M2, M3. While each algorithm will find a maximal set of independent columns, the numerical rank produced by $M 1$ may differ from that produced by. M2, M3. Even if the rank is the same, the maximal sets may contain different colums.

Four tests were performed. In each trial an MxN matrix $A=\left(a_{i j}\right)$ was constructed with $N \in[10,50], M \in[N, 2 N]$, and $a_{1 j} \in[-1,1]$ (each uniformly distributed). In the first test 2000 trlals were run wth relative uncertainty $\gamma_{j}=10^{-4}, j=1, \ldots, N$ and absolute uncertainty $\delta_{j}=10^{-4}, j=1, \ldots, N$. As might be expected, none of the trials produced a rank deficient matrix. Both M2 and M3 computed a total of 4 exact values of p. Figure 5.1 shows the distribution of $\frac{\left|\tilde{\rho}_{j}\right|}{p_{j}}$ and $\frac{\rho_{j}}{\bar{p}_{j}}$ for those trials in which M1 produced the same pivot order as M2, M3. The left half of Figure 5.1 shows the percent of the total with $\frac{\left|\tilde{\rho}_{j}\right|}{\rho_{f}} \times$ and the right half showe the percent of the total with $\frac{\rho_{j}}{\bar{\rho}_{j}} ; x_{i}$ 
In the second test 500 matrices were generated. Each matrix was given the four valves $\gamma_{j}=\delta_{j}=10^{-2}, 10^{-4}$, $10^{-6}, 10^{-8}, j=1, \ldots, N$ for a total of 2000 trials. This test produced 1977 trials of full rank. Of the 23 rank deficlent tr1als, M1 found the same maximal set as M2, M3 in 6 trials and the same rank in 19 trials. Method M2 computed 625 exact values of $\rho$ while M3 computed 620 . Figure 5.2 shows the distribution of $\frac{\tilde{\rho}_{j} \mid}{\rho_{j}}$ and $\frac{\rho_{j}}{\bar{\rho}_{j}}$ for those trials with identical pivot orders.

In the third test, after $N$ and $M$ were chosen, an integer $K$ was chosen so that $N-K<5$ and A was constructed so that the last $\mathrm{N}-\mathrm{K}$ columns were linear combinations of the first $\mathrm{K}$ columns. Then a matrix $\psi=\left(\psi_{1 j}\right)$ with $\psi_{1 j} \varepsilon\left[-10^{-5}, 10^{-5}\right]$ was added to A. Each matrix was given the four valves $\gamma_{j}=\delta_{j}=10^{-2}$, $10^{-4}, 10^{-6}, 10^{-8}, j-1, \ldots, N$ as in the second test. A total of 2000 trlals were run. Only 896 trials were of full rank. of the 1104 rank deficient trials, M1 found the same maximal set as M2, M3 in 951 trials and the same rank in 1078 trials. Method M2 computed 1495 exact values of $p$ while M3 computed 1313. Figure 5.3 shows the distribution of $\frac{\left|\ddot{\rho}_{j \mid}\right|}{\rho_{j}}$ and $\frac{\rho_{j}}{\overline{\rho_{j}}}$ for those trials with identical pivot order.

In the fourth test $A$ was chosen as in the first two tests, but relative and absolute uncertainties were chosen so that $r_{j}=10^{-N E X}$, NEX $\varepsilon[0,14]$, and $\delta_{j} \in[0,1]$ (uniformly distributed). Of 2000 trials only 101 were of full rank." of the 1899 rank deficient trials $M 1$ and $M 2, M 3$ found the same maximal set in 767 and the same rank in 1345. Method M2 computed 17,465 exact values of $\underline{\rho}$ while M3 computed 16,711 . Figure 5.4 shows 


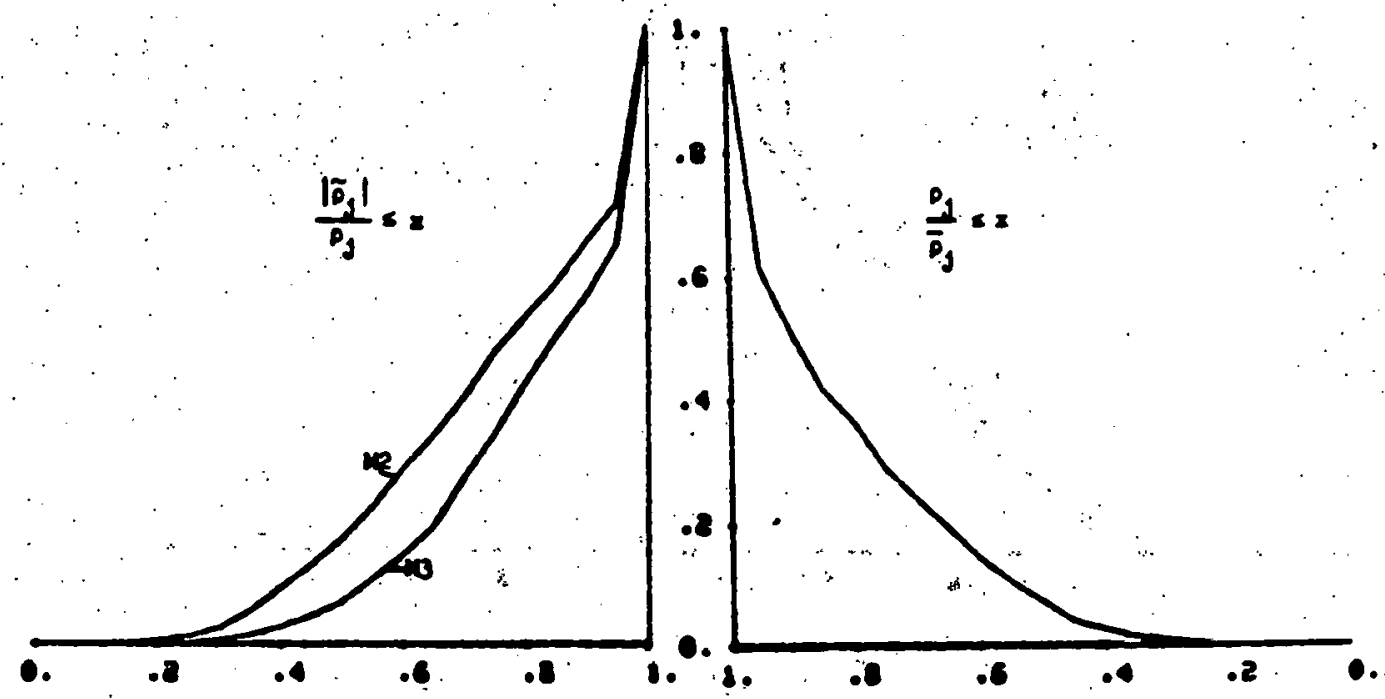

Preire 3.1

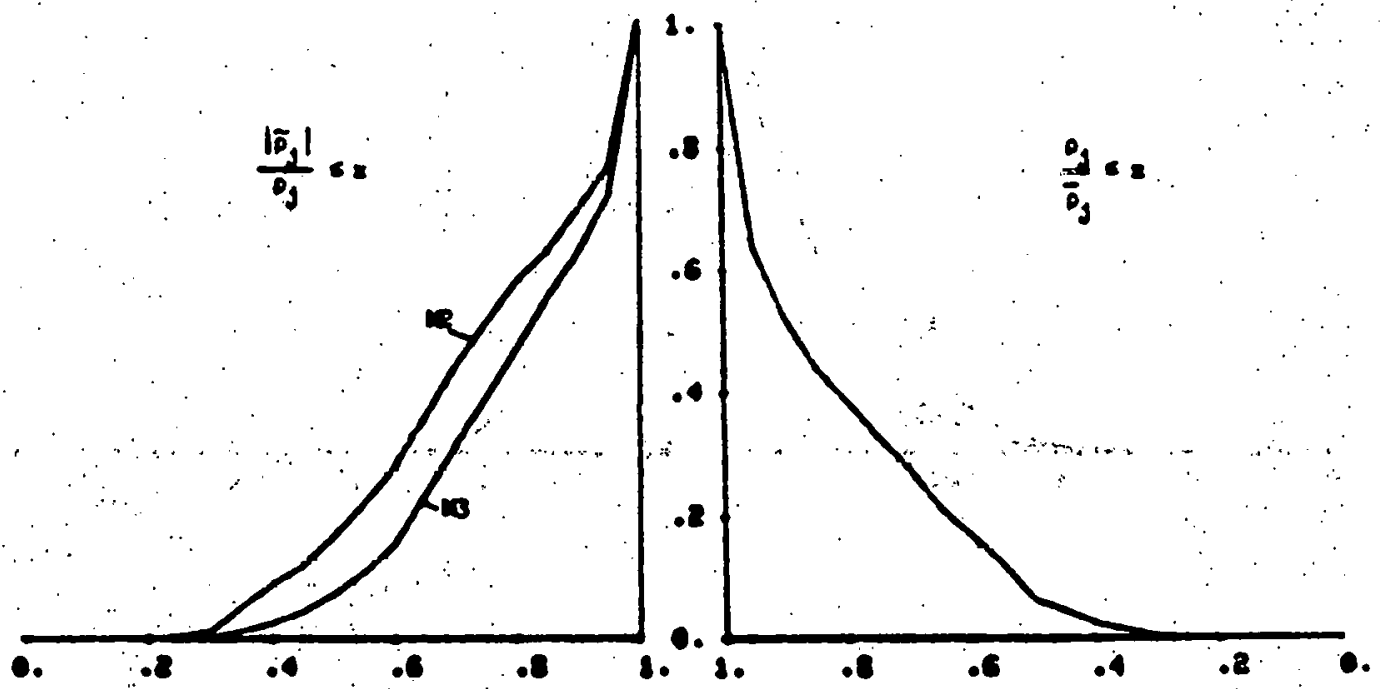

Mare 5.2 


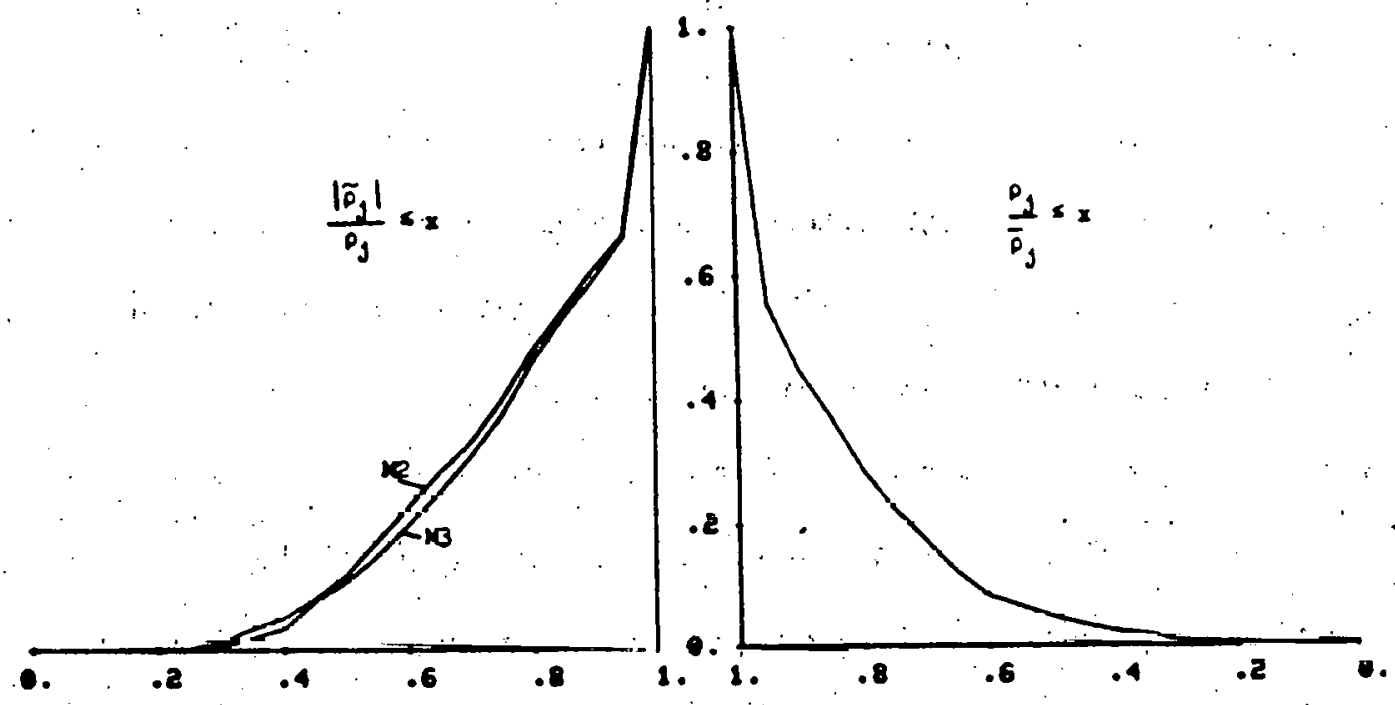

. Moure 5.3

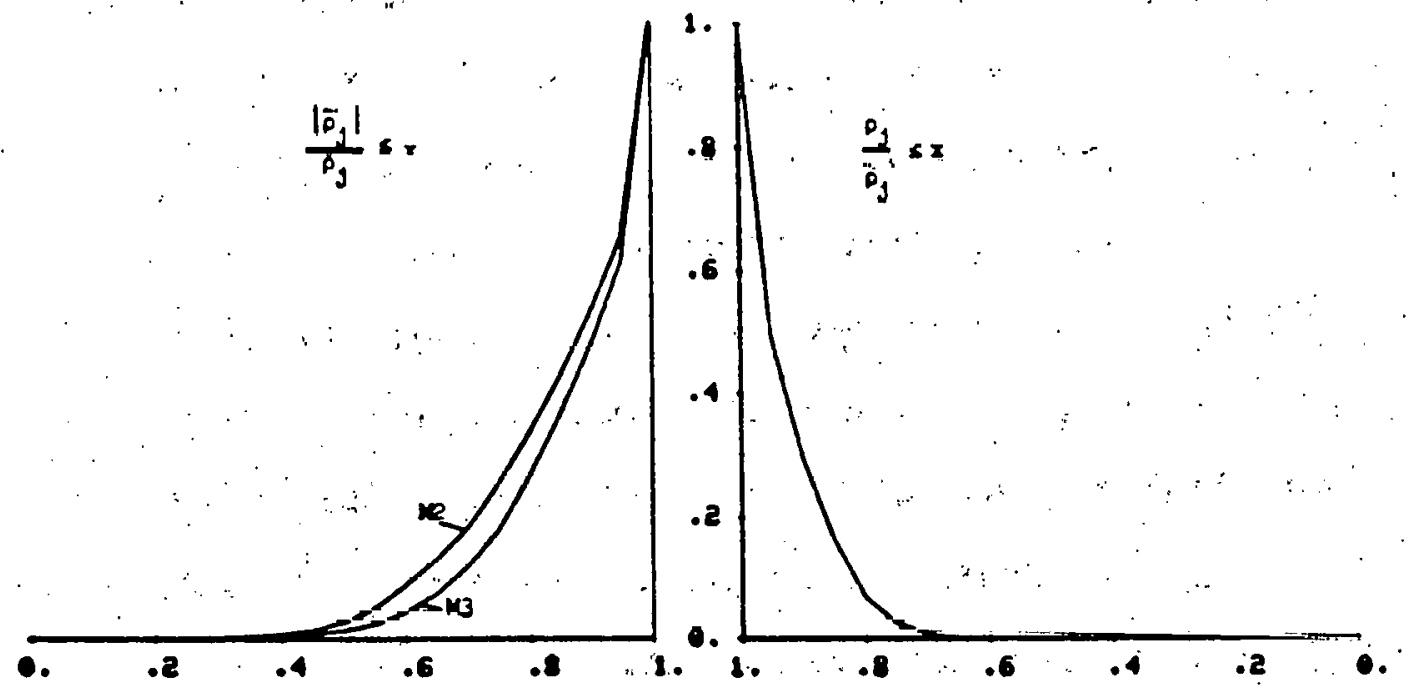

Meure 5.4

32 
the distribution of $\frac{\left|\widetilde{\rho}_{\mathbf{j}}\right|}{\rho_{\mathbf{j}}}$ and $\frac{\rho_{\mathbf{j}}}{\bar{\rho}_{\mathbf{j}}}$ for those trials with identical pivot orders.

The first test is perhaps the most realistic set of

problems. These algorithms were designed to handle problems

that are assumed to be of full rank, wh th proper detection

of that rare instance when rank deficiency occurs. Tests 2,

3 , and 4 we re constructed to produce increasingly more trials

which are rank deficient and rank deficiencies of higher orders.

In test 4 the absolute uncertainty is of the same magnitude as

the elements of $A$.

We may conclude that there is enough similarity between

the performance of algortthms $M 1$ and $M 2, M 3$, in terms of finding a maximal set, that the extra computation required by M1 may be unwarranted. Method MI does provide exact values for $C_{1}(R)$ and $C_{\infty}(R)$, but Figures $5.1-5.4$ show that $M 2$ and $M 3$ also provide

good bounds for $C_{1}(R)$. These bounds are best on the trials

that are rank deficient or nearly rank deficient. Method Ml does provide bounds on $\underline{x}^{I}$ which cannot be extracted from M2 or M3.

The test results also show that $M 2$ is more economical than

M3. While Figures 5.1 - 5.4 show that M3 yields better lower bounds, more artthmetic was required to achleve the same results In terms of finding a maximal set.

In every trial in the first three test we had $k_{1}=k_{2}$ so there was no doubt about the rank of $A_{2}^{I}$. In the fourth test there was uncertalnty as to the rank in some trlals but $k_{2}-K_{1}$ never exceeded 3 . In addition, the length of the shortest least squares solution and the length of the resldual vector produced by $M 1$ were compared to 
the corresponding values produced by the pseudo-inverse of the same rank. A singular value decomposition was used to construct the pseudo-inverse. As might be expected both palr's of values agreed to within the relative uncertainty used to compute the numerical rank with Ml, sometimes many orders of magnitude less. This Indicates that $\mathrm{Ml}$ is producing a good approximation to the pseudoinverse.

Finally, Ml produced a column order in which $\rho_{j}<\rho_{j+1}$, $1=1, \ldots, N^{-1}$ in $95 \%$ of the 8000 , trials. 


\section{References}

[1] N. Anderson and I. Karasalo, "On Computing Bounds for the Least Singular Value of a Triangular Matrix," BIT 15, 1-4, (1975).

[2] P. Businger and G. Golub, "Lịnear Least Squares by Householder Trans formations" Numer. Math. 7, 269-276, (1965).

[3] A. K. Cline, C. B. Moler, G. W. Stewart, and J. H. Wilkinson, "An Estimate for the Condition Number of A Matrix," SIAM J. Numer. Anal., Vol. 16, No. 2, 368-375, (1979).

[4] G. Golub and W. Kahan, "Calculating the Singular Values and Psuedo-Inverse of a Matrix," SIAM J. of Numer. Anal., Ser. B, Vol. 2, No. 2, 205-224 (1965).

[5] G. Golub, "Numerical Methods for Solving Linear Least Squares Problems," Numer. Math. 7, 206-216, (1965).

[6] G. Golub, V. Klema, G. W. Stewart, "Rank Degeneracy and Least Squares Problems," Stanford Report, Stan-CS-76-559, Sțanford University, (1976).

[7]. E. Hanson, "Interval Arithmetic in Matrix Computations, Part I," SIAM J. Numer. Ana1., Vol. 2, No. 2, 308-320, (1965).

[8]: E. Hanson and R. Smith, "Interval Arithmetic in Matrix Computatlons, Part II," SIAM J. Numer. Anal., Vol. 4, No. 1, 1-9,"(1967).

[9] I. Karasalo, "A Criterion for Truncation of the QR-Decomposition Algorfthm for the Singular Least Squares Problem," BIT 14, 156-166, (1974).

[10] C. Lawson and R. Hanson, Solving Least Squares Problems; PrenticeHa11, Englewood Cliffs, NJ, (1974). 
[11] T. A. Manteuffel, "Numerical Rank Determination in Linear. Least Squares Problems;" Sandla Laboratories Report, SAND79-8243, (August 1979).

[12] R: E. Moore; Interval Analysis, Prentice-Hall, Englewood C11ffs, NJ, (1966).

[13]. G. W. Stewart, Introduction to Matrix Computation, Academic Press, New York, (1973).

[14] M. J. D. Powell and J. K. Reid, "On Applying Householder Transformations to Linear Least Squares Problems," Proc:" IF IP 1968 Vol. 1, 122-126, (1969). 
Distribution:

H. van der Vorst

Academic Computer Center Utrecht Budapestlaan 6

De Uithof-Utrecht

The Netherlands 030531436

L. W. Ehrlich

Applied Physics Laboratory

The Johns. Hopkins University

Johns Hopkins Road

Laurel, MD 20810

Dr. John K. Reid

Computer Science System Division

Building 8.9 AERE

Harwell, Didcot

England OXII ORA

Professor Richard S. Varge

Department of Mathematics

Ristit State University

Kent, OH 44242

James C. T. Pool

BR-17, J-309, GTN

Basic Energy Sciences

Department of Energy

Weshington, DC 20545 .

B. I. Buzbee, C-3

Los Alamos Sclentlfic Laboratory

P. O. B Box 1663

Los Alamos, IMM. 87545

Paul Concus

University of California

Lawrence Berkeley Laboratopy

5DA-2129

Berkeley, CA 94720

I. Karasallo

University of Callfornis

Lawrence Berkeley Laboratory

5DA-2129

Berkeley, CA 94720

J. Dongarra

Applied Mathematics Department

Argonne National Laboratorles

9700 S. Cass Avenue

Argonne, II 60439
F. N. Fritsch, $\mathrm{L}-300$

Lawrence If vermore Laboratory

University of California

P. 0. Box 808

Livermore, CA 94550

A. Greenbaum; I-300

Lawrence Livermore Laboratory

University of California

P. 0. Box 808

Livermore, CA 94550

G. Rodrique

Lawrence Livermore Laboratory

University of Caiffornia

P. 0. Box 808

Livermore, CA 94550

G. H. Golub

Dept. of Computer sclence

Stanford University

Stanford, CA 94305

R. Sweet

National Center for Atmospheric Research P. 0. Box 3000

Boulder, CO 80303

Paul Saylor

Department of Computer Science

University of Illinois.

Urbana, II 61820

Professor Cleve B. Moler

Department of Mathematics

University of New Mexico

Albuquerque, IM 87106

2620. R. J. Detry

2646 M. R. Scott

2646 K. Haskell

2646 H. Watts

3141 T. I. Werner (5)

3151 W. I. Garner (3)

For DOE/TIC (Unlim. Release)

3154-3 R. P. CampbeII (25) (For DOE/TIC)

5600 D. B. Shuster

Attn: 5610 A. A. Iieber

5620 M. M. Newsom

5630 R. C. Maydew

5640 G. J. Simmons 
5641 R. J. Thompson

5642 L. F. Shampine

5642 R. J. Hanson

5642 K. L. Hiebert

5642

8000

T. A. Manteuffel (25)

T. B. Cook

Attn: 8100 W. J. Spencer

8200 A. N. Blackwell

8300 B. F. Murphey

8400 L. Gutierrez

8330 G. W. Anderson

8331 R. J. Kee

8332 R. E. Huddleston

8332 R. E. Chang

8332 D. L. Crewford

8332 T. II. Jefferson

8333 A. G. Schuknecht

835 li B. Senders. 
